\title{
ON KINETIC APPROACH TO MODELING OF SOURCES OF ELECTROMAGNETIC RADIATION LOCATED IN PLANET/STELLAR ELECTROMAGNETIC STRUCTURES
}

\author{
V. M. Gubchenko*
}

\begin{abstract}
Electromagnetic radiation from stars and planets appeared at the different wavebands. Radiation is provided by accelerated particles originated from plasma electromagnetic structures which are named the particle sources. Structures are selfconsistent with inductive e.m. fields and collisionless hot plasma electrodynamics including plasma part formed by accelerated particles. Here quasifree acceleration by inductive eddy e.m. field takes place. The sources are related with the diffusion regions (DR) of plasma where we get weak particle magnetization and where plasma kinetic process of magnetic reconnection takes place. In the DR electric current can be parted on the diamagnetic and on the resistive components. Current components in DR source are in ratio $\mathrm{G}$ and the components are related with non accelerated (diamagnetic) particles and accelerated (resistive) particles. Parameter G is calculated via momentum and energy anisotropy parameters depending on shape of the particle velocity distribution function (VDF). Parameter $\mathrm{G}$ can be positive and negative; $\mathrm{G}$ defines resistive, diamagnetic and quasi-current free dynamics of the $\mathrm{DR}$ as a source of accelerated particles. The $\mathrm{G}$ is the e.m. analog of the acoustic Mach number M. Plasma DR structures are described by anomalous skin-scale and diamagnetic skin scale. We introduced criteria on value of plasma beta and on value of anisotropy parameters to get the DR plasma structures realization.
\end{abstract}

\footnotetext{
* Institute of Applied Physics, Russian Academy of Science, 46 Ul'yanov Street, 603950, Nizhni Novgorod, Russia
} 
